\title{
Shared Genetic Links Between Amyotrophic Lateral Sclerosis and Obesity-Related Traits: A Genome- Wide Association Study
}

\section{Chunyu Li}

Sichuan University West China Hospital Department of Neurology

\section{Ruwei Ou}

Sichuan University West China Hospital Department of Neurology

\section{Xiaojing Gu}

Sichuan University West China Hospital

\section{Qianqian Wei}

Sichuan University West China Hospital

\section{HuiFang Shang ( $\nabla$ hfshang2002@126.com )}

Sichuan University West China Hospital Department of Neurology https://orcid.org/0000-0003-09471151

\section{Research article}

Keywords: amyotrophic lateral sclerosis, obesity, pleiotropy, genome-wide association study

Posted Date: August 12th, 2020

DOl: https://doi.org/10.21203/rs.3.rs-50516/v1

License: (c) (i) This work is licensed under a Creative Commons Attribution 4.0 International License. Read Full License

Version of Record: A version of this preprint was published at Neurobiology of Aging on June 1st, 2021. See the published version at https://doi.org/10.1016/j.neurobiolaging.2021.01.023. 


\section{Abstract}

Background: Epidemiological and clinical studies have suggested comorbidity between amyotrophic lateral sclerosis (ALS) and obesity-related traits. However, little is known about their shared genetic architecture.

Objective: To examine whether there exist genetic enrichment between ALS and eleven obesity-related traits, including body mass index (BMI), waist hip ratio, body fat percentage (BFP), birth weight, triglycerides, total cholesterol, low-density lipoprotein cholesterol (LDL-C), high-density lipoprotein cholesterol (HDL-C), type 2 diabetes (T2D), fasting glucose, fasting insulin, and identify shared loci among them.

Methods: Using the conditional false discovery rate (FDR) statistical framework, we analyzed genome wide association summary statistics for ALS $(n=80610)$ and obesity-related traits, and further conducted functional enrichment analysis.

Results: Robust genetic enrichment was observed for ALS conditional on BMI, BFP, HDL-C, LDL-C and T2D, but minimal enrichment on the other traits. 9 shared genetic loci with conjunctional FDR $<0.05$ was identified, among which 6 were replicated in a second ALS cohort, including rs3849942 (C9orf72), rs170663 (G2E3), rs8018993 (SCFD1), rs978220 (ATXN3), rs62333164 (CLCN3) and rs12603276 (GGNBP2). We further identified GGNBP2 as a novel potential ALS risk gene, by integrating cis-expression quantitative trait loci analysis in human brain tissue and summary-data-based Mendelian randomization analysis. Functional analysis indicated the shared risk genes were involved in pathways membrane trafficking and vesicle-mediated transport.

Conclusions: Our findings demonstrate selective genetic overlap between ALS and obesity-related traits, and identified new shared risk loci, including novel potential ALS risk gene GGNBP2. These results provide better understanding for the pleiotropy of ALS and have implications for future therapeutic trials.

\section{Background}

Amyotrophic lateral sclerosis (ALS) is a fetal neurodegenerative disorder with an average survival period around 3-5 years since the symptom onset, which brings a substantial impact on the quality of life for patients and their families, and huge socioeconomic burdens[1,2]. With the advent of next-generation sequencing, emerging evidence suggests a substantial genetic component underlying ALS[3, 4]. Large genome-wide association studies (GWAS) have identified a growing number of susceptibility loci related to ALS and provided novel insight into the pathogenesis of ALS[5, 6]. However, due to the polygenic architecture of ALS, risk loci with weak associations cannot be identified with current GWAS sample size[7]. There is still a large "missing heritability" to be discovered, with an estimated heritability by twin data as high as $65 \%[8]$, while just $21 \%$ by current risk loci[9]. Moreover, some of the nominated risk loci have not been implicated in disease pathogenesis and are awaiting further exploration. 
The association between ALS and obesity-related traits has long been observed. ALS patients often encounter a loss of weight or a decreased body mass index (BMI) and body fat along with disease progression[10,11]. Dyslipidemia is also a quite common characteristic of ALS patients, and higher levels of total cholesterol (TC), low-density lipoprotein cholesterol (LDL-C), total triglycerides (TG), and LDL$\mathrm{C} /$ high-density lipoprotein cholesterol (HDL-C) ratio have been shown to be more prevalent in ALS patients than in controls, although subsequent results are not consistent[12-14]. The polygenic risk scores based on LDL-C and TC risk alleles are also shown to be associated with ALS risk[15]. Besides, a protective role of type 2 diabetes (T2D) on ALS has been found in observational studies[16-18]. These clinical and epidemiological findings suggest the obesity-related traits may potentially possess shared genetic background with ALS[19], but to what extent they overlap has not been investigated yet.

Recently, a novel statistical method to investigate overlapping between polygenic traits using GWAS summary statistics has been developed, and utilized in several human traits and diseases[20-23]. By incorporating GWAS results from multiple disorders and phenotypes, this method could provide insights into genetic pleiotropy (defined as a single gene or variant being associated with more than one distinct phenotype) and increase statistical power to discover significant associations[20-22]. Applying this approach, we systematically evaluated shared genetic risk between ALS and eleven obesity-related traits, including obesity traits like BMI, waist hip ratio (WHR), body fat percentage (BFP) and birth weight (BW), obesity-related lipid traits like LDL-C, HDL-C, TC and TG, and obesity related glucose traits like T2D, fasting glucose $(\mathrm{FG})$ and fasting insulin (FI). We further replicated the shared risk loci in a second ALS cohort, and conducted functional enrichment analysis with the identified shared risk genes.

\section{Methods}

\section{GWAS summary statistics}

We investigated the shared genetic architecture between ALS[6] and obesity-related traits including BMI[24], WHR[25], BFP[26], BW[27], HDL-C, LDL-C, TC, TG[28], FI, FG[29] and T2D[30] based on GWAS summary statistics. Details of the summary data from all GWAS used in the current study were listed in SupplementaryTable 1, and the study design including collection of samples, quality control procedures, imputation methods etc. for each GWAS have been described in each publication. To confirm the findings in the discovery phase, we further assessed the $\mathrm{P}$ values of the identified pleiotropic single nucleotide polymorphisms (SNP) in a second ALS cohort of mixed ancestries[31] $(n=41395)$. If the identified pleiotropic SNP was not in the replication GWAS, the nearest proxy SNP in strong linkage disequilibrium (LD) $\left(R^{2}>0.8, D^{\prime}>0.8\right)$ was chosen instead. The relevant institutional review boards or ethics committees approved the research protocol of each GWAS used in the current analysis, and all human participants gave written informed consent.

\section{Genomic control}

Due to population stratification or cryptic relatedness or overcorrection of test statistics[32], the empirical null distribution in GWAS is sometimes inflated or deflated. To correct for such bias, we computed the 
genomic inflation factor $\lambda_{G C}$ using intergenic SNPs, and adjusted the summary statistics by $\lambda_{G C}$ for each GWAS respectively. Briefly, we annotated each SNP with ANNOVAR[33] and filtered intergenic SNPs only, since they provide a robust estimate of the true null effects[34]. Then we estimated $\lambda_{G C}$ as the median $Z$ score squared divided by the expected median of a chi-square distribution with one degree of freedom. After genomic control adjustment, we pruned the SNPs by removing SNPs in LD ( $r^{2}>0.2$ within $\left.250 \mathrm{~kb}\right)$ based on 1000 Genomes Project LD structure.

\section{Genetic enrichment}

Quantile-quantile plots compare a nominal probability distribution against an empirical distribution, and leftward deflection of the observed distribution reflect enrichment of low $P$ values. To assess the pleiotropic enrichment, we plotted conditional quantile-quantile plot for a primary phenotype by creating subsets of SNPs based on their association with a second phenotype. Specifically, we computed the empirical cumulative distribution of nominal $P$ values of ALS for all SNPs and for subsets of SNPs with significance level below the indicated cutoffs on another trait $\left(-\log _{10}(P) \geq 0,-\log _{10}(P) \geq 1,-\log _{10}(P) \geq 2\right.$, $\log _{10}(P) \geq 3$, corresponding to $\left.P \leq 1, P \leq 0.1, P \leq 0.01, P \leq 0.001\right)[20,34]$.

Furthermore, to assess the level of enrichment, we constructed fold-enrichment plots of nominal $-\log _{10}(P)$ values of ALS for all SNPs and subsets of SNPs determined by the significance of their association with each obesity-related trait. The presence of enrichment is reflected as an upward deflection of the curve for the primary phenotype, and the degree of deflection depends on the degree of association with the second phenotype. Enrichment can be directly interpreted in terms of the true discovery rate, which is equal to 1 - false discovery rate (FDR). To assess polygenic effects, we focused on SNPs with nominal $\log _{10}(P)<7.3$ (corresponding to $\left.P>5 \times 10^{-8}\right)[20,34]$.

\section{Identification of risk loci}

To identify risk loci associated with ALS conditional on each obesity-related trait, we computed the conditional FDR statistics using the stratified FDR approach[20-23]. The FDR framework is based on Bayesian statistics, which incorporates information from GWAS summary results of a second phenotype to adjust its original significance level. The conditional FDR is the probability of the SNP being null given its $P$ value is as small as or smaller than observed. To remove false positives, a significance threshold of FDR $<0.01$ was utilized. Conditional Manhattan plot was created to illustrate the genetic markers associated with ALS conditional on each trait[20,34].

Furthermore, to identify shared risk loci associated with ALS and each obesity-related trait, we computed the conjunctional FDR statistics. The conjunctional FDR is an extension of the conditional FDR and is defined as the maximum of the two conditional FDR statistics for a specific SNP. Conjunctional FDR estimates the posterior probability that a SNP is null for both traits simultaneously, given that the $P$ values for both phenotypes are smaller than observed respectively. A significance threshold of FDR $<0.05$ was utilized, corresponding to five false positives per 100 reported associations. Conjunctional 
Manhattan plot was created to illustrate the shared genetic markers associated with ALS and each trait[20,34].

\section{Functional evaluation of shared risk loci}

To assess whether the shared risk loci modify gene expression, we evaluated cis-expression quantitative trait loci (eQTL) in Braineac, a public dataset of normal control brains for investigating the genes and SNPs associated with neurological disorders[35]. We analyzed eQTL for the mean P value derived across these brain regions: the cerebellum, frontal cortex, hippocampus, medulla, occipital cortex, putamen, substantia nigra, temporal cortex, thalamus, and white matter. To minimize false positives, a $P$ value below 1.5E-03 was considered significant after Bonferroni correction.

To identify enrichments in gene ontologic (GO) features associated with ALS and obesity-related traits, we used ConsensusPathDB[36] for functional interaction analysis, which compare $\mathrm{GO}$ terms between background and candidate gene sets using the hypergeometric test and generates $\mathrm{P}$ values corrected for multiple testing. The shared risk genes identified with conjunctional FDR method and QQTL analysis were utilized with default parameters and default background gene set. Biological, cellular, and molecular GO terms were analyzed.

\section{Results}

\section{Estimation of pleiotropic enrichment}

In the stratified quantile-quantile plots for ALS conditional on association P values with each obesityrelated trait, successive enrichment was found for BMI, BFP, HDL-C, LDL-C and T2D (Figure 1), indicating the proportion of non-null SNPs in ALS increase with higher levels of association with these traits. In contrast, minimal or no enrichment was found for WHR, BW, TC, TG, FG and FI. For progressively stringent $P$ value thresholds, we could observe approximately 13-fold enrichment conditional on BMI, 13-fold enrichment on BFP, 10-fold enrichment on LDL-C, 8-fold enrichment on HDL-C and 8-fold enrichment on T2D, while minimal enrichment on the other traits, suggesting selective genetic overlap between ALS and obesity-related traits (Figure 2).

\section{ALS-associated loci identified with conditional FDR}

To discover genetic variants associated with ALS, we performed the conditional FDR statistical analysis. A total of 20 risk loci were identified with conditional FDR $<0.01$ (SupplementaryTable 2), including 14 novel loci which were not significant $(P<5 E-08)$ in the original ALS GWAS[6]. In these 20 loci, 11 were suggestively significant $(\mathrm{P}<1 \mathrm{E}-04)$ in the replication GWAS results, namely rs10463311 (GPX3,TNIP1), rs7813314 (LOC101927815), rs7864502 (C9orf72), rs58854276 (ACSL5), rs12810996 (TBK1), rs447614 (G2E3), rs229150 (SCFD1), rs978220 (ATXN3), rs35714695 (SARM1), rs2285642 (GGNBP2) and rs12608932 (UNC13A) (SupplementaryTable 2). Among these replicated genes, GPX3, TNIP1, C9orf72, 
TBK1, SCFD1, ATXN3 and UNC13A have been described as risk genes for ALS by earlier GWAS, while the others were novel risk genes, including LOC101927815, ACSL5, G2E3, GGNBP2 and SARM1.

\section{Loci shared between ALS and obesity-related traits}

To identify shared risk loci between ALS and obesity-related traits, we further performed conjunctional FDR analysis. A total of 9 shared risk loci were identified with conjunctional FDR $<0.05$, including rs62333164 (CLCN3), rs170663 (G2E3), rs8018993 (SCFD1), rs11160036 (TRIP11), rs978220 (ATXN3) and rs12603276 (GGNBP2) between ALS and obesity traits BMI/WHR/BFP, rs3849942 (C9orf72) and rs1976704 (PGS1) between ALS and HDL-C/LDL-C, and rs68069258 (DENND6B) between ALS and T2D (Table 1). In these 9 risk loci, 6 were suggestively significant $(P<1 \mathrm{E}-04)$ in the replication GWAS results, namely rs62333164 ( $P=3.84 E-06)$, rs3849942 ( $P=1.20 E-22)$, rs170663 ( $P=2.22 E-06)$, rs8018993 ( $P=8.00 E-$ 06), rs978220 ( $P=2.61 \mathrm{E}-05)$ and rs12603276 ( $\mathrm{P}=9.21 \mathrm{E}-06)$ (Table 1). Among these replicated genes, $C L C N 3, G 2 E 3$, and GGNBP2 were newly discovered risk genes for ALS, while the others have been described as risk genes for ALS by previous GWAS. No shared risk loci were found for ALS conditional on TC, TG, FG, FI and BW, which was consistent with the stratified quantile-quantile plots and foldenrichment plots with no apparent enrichment observed. Notably, in the 6 shared risk loci identified between ALS and BMI, 5 were in reverse effect direction for ALS and BMI in the original GWAS. This is in line with recent Mendelian randomization results[37] that premorbid higher BMI contributes to decreased ALS risk. In contrast, the effect direction of the two shared risk SNPs between ALS and HDL-C were not consistent.

\section{Functional interpretation of shared risk loci}

To determine the functional effects of the shared risk loci, we evaluated cis-eQTL in human brains free of neuropathologic characteristics. The pleiotropic risk SNPs in GGNBP2 were associated with expression of MY019 ( $P=3.10 \mathrm{E}-04)$ and GGNBP2 $(P=8.40 \mathrm{E}-12)$, both of which were risk genes associated with BMI. Pleiotropic SNPs in C9orf72 affect expression of TEK ( $\mathrm{P}=3.40 \mathrm{E}-05)$, which is also associated with blood protein level. Pleiotropic SNPs in ATXN3 were related to expression of SLC24A4 ( $\mathrm{P}=1.10 \mathrm{E}-03)$ and ATXN3 $(\mathrm{P}=1.10 \mathrm{E}-06)$, particularly in cerebellum. Interestingly, SLC24A4 is risk gene for Alzheimer disease and $A T X N 3$ is risk gene for ALS, suggesting this region might have some common effect in neurodegenerative diseases. In addition, pleiotropic SNPs in PGS1 affect expression of PGS1 ( $P=6.60 \mathrm{E}-11)$ and USP36 ( $P=2.00 \mathrm{E}-04)$ (Table 2).

To determine the biological pathways represented by shared risk genes and the genes identified with eQTL analysis, we conducted pathway overrepresentation analysis. Two pathways were enriched, namely membrane trafficking $(P=0.004)$ and vesicle-mediated transport $(P=0.005)$, both of which have been identified as involved in the pathogenesis of ALS. Membrane trafficking has been implicated in virtually every aspect of neuronal function and, in particular, neuronal maintenance and degeneration[38], and intracellular membrane trafficking defects affecting key neuronal functions may be an early determinant of motor neuron loss in ALS[39]. Meanwhile, the molecular regulation of intracellular and extracellular vesicle trafficking is an important pathway in ALS pathogenesis[40], and mutation in the vesicle- 
trafficking protein has been implicated to cause late-onset spinal muscular atrophy and amyotrophic lateral sclerosis. Additionally, $7 \mathrm{GO}$ sets were identified, most of which are related to the membrane trafficking and cytoskeleton (SupplementaryTable 3).

\section{Discussion}

Using summary statistics form large GWAS and conditional FDR statistical framework, we investigated genetic enrichment between ALS and 11 obesity-related traits. Genetic enrichment was observed for ALS conditional on BMI, BFP, HDL-C, LDL-C and T2D, while not on the others. Totally, we identified 9 shared risk loci between ALS and these traits in the discovery phase, with 6 validated in the replication GWAS, and rs12603276 (GGNBP2) was further annotated as related to gene expression in human brain tissue by eQTL analysis. This is the first time that pleiotropic enrichment was systematically assessed between ALS and obesity-related traits. Our findings suggest the polygenic component in ALS is enriched in obesity-related traits, and the enrichment varies between different traits.

We observed obvious enrichment for ALS as a function of BMI and BFP, but not BW, which was consistent with previous epidemiologic evidence[41, 42]. For example, lower BMI or quick BMI decrease has been observed associated with short ALS survival[41, 42]. A prospective study on 518,108 individuals found that increased pre-diagnostic body fat is associated with a decreased risk of ALS mortality. In contrast, little evidence was found for the association between ALS and BW, in line with the notion that ALS is a neurodegenerative disease, rather than neurodevelopmental disease. Our findings demonstrated correlation between ALS and obesity traits from a genetic perspective. In the shared risk genes identified using obesity traits, SCFD1 and ATXN3 have been reported associated with ALS by GWAS[6]. It is noteworthy that both of these two genes were involved in regulation of protein processing, transport and metabolism, and abnormal protein metabolism has been observed in both ALS and obesity[43]. Therefore, these two genes might act as genetic links between ALS and obesity through these cellular processes.

Moreover, we identified a novel risk locus rs12603276 (GGNBP2) for ALS conditional on BMI, WHR and BFP, and validated this locus as cis-eQTL for GGNBP2 in human brain tissue. Previous studies have also suggested that GGNBP2 might be associated with ALS through gene-based association analysis and summary statistics-based Mendelian randomization (SMR) analysis[31, 44]. Our findings demonstrate that GGNBP2 may be a link between ALS and obesity. Meanwhile, we noticed that GGNBP2 is an important tumor suppresser involved in several kinds of cancers[45]. Cancer and neurodegenerative diseases are often hailed as the two sides of a coin[46]. As is observed in epidemiological studies, the overall risk of cancer was significantly reduced in cases with ALS[47]. The pathways that cause neuronal apoptosis, like mitogen-activated protein kinase (MAPK) signaling, can cause uncontrolled neuronal growth as well[48]. GGNBP2 might act as potential genetic links between cancer and ALS. Based on joint evidence from genetic enrichment, $\mathrm{QQTL}$ and SMR analyses, GGNBP2 might be risk gene for ALS and is worth further functional exploration. 
In clinical practice, dyslipidemia has been suggested to be associated with ALS, and hypercholesterolemia was initially associated with lower risk of ALS[49]. However, contradictory evidence has also been presented, and several studies have shown conflicting results on whether the levels of HDLC, LDL-C or TC were different between ALS patients and controls[50, 51]. In our study, enrichment was found for ALS conditional on HDL-C and LDL-C, but minimal or no enrichment on TC and TG. In addition, C9orf72 was identified as a shared risk gene for ALS and HDL-C/LDL-C. C9orf72 repeat expansion was a common cause for ALS, especially in the Caucasian population. The pathogenesis of C9orf72 is still debated, with proposed mechanisms including repeated RNA-mediated toxicity, dipeptide protein toxicity or haplodeficiency[52]. Recently, decreased total serum HDL-C concentration level was observed in C9orf72 repeat expansion carriers, suggesting that the pathogenic mechanism of C9orf72 repeated expansion mutation may be related to abnormal lipid metabolism[53]. Our findings further confirmed that C9orf72 is an important gene coupling ALS and lipid metabolism from a genetic perspective. The underlying mechanism of $C 9$ orf 72 in the lipid metabolism changes and ALS pathogenesis was worth further exploration.

Previous studies have suggested that T2D has a protective effect for ALS in case-control studies[54, 55]. In the current study, we found enrichment for ALS conditional on T2D, but not FI and FG. Lack of enrichment on FI and FG suggests the origin of glucose homeostasis abnormalities in ALS may be multifactorial, and other pathways might explain the enrichment for T2D. For example, our previous study found that higher levels of $\mathrm{HbA1c}$, but not fasting blood glucose concentrations, were significantly associated with higher risks of ALS mortality[56]. Additionally, we identified one shared risk gene DENND6B between ALS and T2D. DENND6B belongs to Differentially Expressed in Normal and Neoplasia (DENN) like families, which is a GDP/GTP exchange factor (GEF) that activates Rab-GTPases[57]. It has been established that $D E N N D 6 B$ is involved in the Rab guanyl-nucleotide exchange factor activity and vesicle-mediated transport, which was suggested to play an important role in ALS[58]. Interestingly, structural prediction engines suggested DENN as a possible homologue of C9orf72, prompting a more complex potential relationship between the DENN family and ALS[59].

There are some limitations of this study. First, the GWASs used in current study were mostly performed on participants of European ancestry, thus the findings of shared genetic architecture might be biased and not applicable to other populations. Future studies in other non-European populations will provide more comprehensive understandings. Second, there were potential sample overlap between ALS and each obesity-related trait in the original GWAS. Such overlap might bring some bias to the statistical analysis, although the bias will be minimal. Third, how the identified pleiotropic SNPs were involved in the pathogenesis of ALS and obesity-related traits cannot be determined. Further functional explorations will provide better understandings.

\section{Conclusions}

By integrating GWAS summary data and conditional FDR statistical framework, we identified selective pleiotropy and novel shared loci between ALS and 11 obesity-related traits. Moreover, we identified a 
novel ALS risk gene GGNBP2 by combining eQTL analysis results. These findings may provide novel insights into the shared genetic background between ALS and obesity-related traits, and help better understand the etiology of ALS and have an impact on the clinical treatment.

\section{Abbreviations}

ALS

amyotrophic lateral sclerosis; $\mathrm{BMI}=$ body mass index; WHR = waist hip ratio; $\mathrm{BFP}$ = body fat percentage; $\mathrm{BW}=$ birth weight; TG = triglycerides; TC = total cholesterol; LDL-C = low-density lipoprotein cholesterol; HDL-C = high-density lipoprotein cholesterol; T2D = type 2 diabetes; FG = fasting glucose; FI = fasting insulin; FDR = false discovery rate; GWAS = genome-wide association studies; SNP = single nucleotide polymorphisms; LD = linkage disequilibrium; eQTL = cis-expression quantitative trait loci; GO = gene ontology; SMR = summary statistics-based Mendelian randomization

\section{Declarations}

\section{Competing interests}

The authors declare that they have no competing interests.

\section{Availability of data and material}

The GWAS summary statistics used to perform the analyses described in the study were obtained from publicly available published data. All data generated or analyzed in the study were included in the article and supplementary material.

Supplemental Data include three tables.

\section{Funding}

This research was supported by the funding of the National Natural Science Foundation of China (Grant No. 81871000), the China Postdoctoral Science Foundation (Grant No. 2019M653424), and the PostDoctor Research Project, West China Hospital, Sichuan University (Grant No. 18HXBH037).

\section{Authors' contributions}

LCY and SHF conceived the study. LCY performed the statistical analyses and prepared the drafted manuscript. ORW and GXJ assisted in the statistical analysis. LCY, ORW, GXJ, WQQ and H.S. contributed to writing and editing of the manuscript. All authors reviewed and approved the final manuscript

\section{Acknowledgements}

We thank the investigators who made the GWAS summary data publicly available and the participants who contributed to those studies. 


\section{References}

1. Adelman EE, Albert SM, Rabkin JG, Del Bene ML, Tider T, O'Sullivan I: Disparities in perceptions of distress and burden in ALS patients and family caregivers.Neurology 2004, 62:1766-1770.

2. Schönfelder E, Osmanovic A, Müschen LH, Petri S, Schreiber-Katz O: Costs of illness in amyotrophic lateral sclerosis (ALS): a cross-sectional survey in Germany.Orphanet journal of rare diseases 2020, 15:149.

3. Turner MR, Hardiman O, Benatar M, Brooks BR, Chio A, de Carvalho M, Ince PG, Lin C, Miller RG, Mitsumoto $\mathrm{H}$, et al: Controversies and priorities in amyotrophic lateral sclerosis. The Lancet Neurology 2013, 12:310-322.

4. Renton $A E$, Chiò A, Traynor BJ: State of play in amyotrophic lateral sclerosis genetics.Nat Neurosci 2014, 17:17-23.

5. van Rheenen W, Shatunov A, Dekker AM, McLaughlin RL, Diekstra FP, Pulit SL, van der Spek RA, Võsa $\mathrm{U}$, de Jong S, Robinson MR, et al: Genome-wide association analyses identify new risk variants and the genetic architecture of amyotrophic lateral sclerosis. Nature genetics 2016, 48:1043-1048.

6. Nicolas A, Kenna KP, Renton AE, Ticozzi N, Faghri F, Chia R, Dominov JA, Kenna BJ, Nalls MA, Keagle P, et al: Genome-wide Analyses Identify KIF5A as a Novel ALS Gene.Neuron 2018, 97:12681283.e1266.

7. McCarthy MI, Abecasis GR, Cardon LR, Goldstein DB, Little J, loannidis JP, Hirschhorn JN: Genomewide association studies for complex traits: consensus, uncertainty and challenges. Nature reviews Genetics 2008, 9:356-369.

8. Al-Chalabi A, Fang F, Hanby MF, Leigh PN, Shaw CE, Ye W, Rijsdijk F: An estimate of amyotrophic lateral sclerosis heritability using twin data.Journal of neurology, neurosurgery, and psychiatry 2010 , 81:1324-1326.

9. Keller MF, Ferrucci L, Singleton AB, Tienari PJ, Laaksovirta H, Restagno G, Chio A, Traynor BJ, Nalls MA: Genome-wide analysis of the heritability of amyotrophic lateral sclerosis.JAMA neurology 2014, 71:1123-1134.

10. Nakken $\mathrm{O}$, Meyer HE, Stigum H, Holmoy T: High BMI is associated with low ALS risk: A populationbased study. 2019, 93:e424-e432.

11. Peter RS, Rosenbohm A, Dupuis L, Brehme T, Kassubek J, Rothenbacher D, Nagel G, Ludolph AC: Life course body mass index and risk and prognosis of amyotrophic lateral sclerosis: results from the ALS registry Swabia.European journal of epidemiology 2017, 32:901-908.

12. Dorst J, Kuhnlein P, Hendrich C, Kassubek J, Sperfeld AD, Ludolph AC: Patients with elevated triglyceride and cholesterol serum levels have a prolonged survival in amyotrophic lateral sclerosis. Journal of neurology 2011, 258:613-617.

13. Chio A, Calvo A, Ilardi A, Cavallo E, Moglia C, Mutani R, Palmo A, Galletti R, Marinou K, Papetti L, Mora G: Lower serum lipid levels are related to respiratory impairment in patients with ALS.Neurology 2009, 73:1681-1685. 
14. Yang JW, Kim SM, Kim HJ, Kim JE, Park KS, Kim SH, Lee KW, Sung JJ: Hypolipidemia in patients with amyotrophic lateral sclerosis: a possible gender difference? Journal of clinical neurology (Seoul, Korea) 2013, 9:125-129.

15. Chen X, Yazdani S, Piehl F, Magnusson PKE, Fang F: Polygenic link between blood lipids and amyotrophic lateral sclerosis.Neurobiology of aging 2018, 67:202.e201-202.e206.

16. Mariosa D, Kamel F, Bellocco R, Ye W, Fang F: Association between diabetes and amyotrophic lateral sclerosis in Sweden.European journal of neurology 2015, 22:1436-1442.

17. Kioumourtzoglou MA, Rotem RS, Seals RM, Gredal O, Hansen J, Weisskopf MG: Diabetes Mellitus, Obesity, and Diagnosis of Amyotrophic Lateral Sclerosis: A Population-Based Study.JAMA neurology 2015, 72:905-911.

18. Zeng $\mathrm{P}$, Wang T, Zheng J, Zhou X: Causal association of type 2 diabetes with amyotrophic lateral sclerosis: new evidence from Mendelian randomization using GWAS summary statistics.BMC medicine 2019, 17:225.

19. Stearns FW: One hundred years of pleiotropy: a retrospective.Genetics 2010, 186:767-773.

20. Andreassen OA, Djurovic S, Thompson WK, Schork AJ, Kendler KS, O'Donovan MC, Rujescu D, Werge $\mathrm{T}$, van de Bunt $\mathrm{M}$, Morris AP, et al: Improved detection of common variants associated with schizophrenia by leveraging pleiotropy with cardiovascular-disease risk factors.American journal of human genetics 2013, 92:197-209.

21. Yokoyama JS, Wang Y, Schork AJ, Thompson WK, Karch CM, Cruchaga C, McEvoy LK, Witoelar A, Chen $\mathrm{CH}$, Holland D, et al: Association Between Genetic Traits for Immune-Mediated Diseases and Alzheimer Disease.JAMA neurology 2016, 73:691-697.

22. Witoelar A, Jansen IE, Wang Y, Desikan RS, Gibbs JR, Blauwendraat C, Thompson WK, Hernandez DG, Djurovic S, Schork AJ, et al: Genome-wide Pleiotropy Between Parkinson Disease and Autoimmune Diseases.JAMA neurology 2017, 74:780-792.

23. Broce IJ, Tan CH, Fan CC, Jansen I, Savage JE, Witoelar A, Wen N, Hess CP, Dillon WP, Glastonbury $\mathrm{CM}$, et al: Dissecting the genetic relationship between cardiovascular risk factors and Alzheimer's disease.Acta neuropathologica 2019, 137:209-226.

24. Yengo L, Sidorenko J, Kemper KE, Zheng Z, Wood AR, Weedon MN, Frayling TM, Hirschhorn J, Yang J, Visscher PM: Meta-analysis of genome-wide association studies for height and body mass index in 700000 individuals of European ancestry.Human molecular genetics 2018, 27:3641-3649.

25. Pulit SL, Stoneman C, Morris AP, Wood AR, Glastonbury CA, Tyrrell J, Yengo L, Ferreira T, Marouli E, Ji Y, et al: Meta-analysis of genome-wide association studies for body fat distribution in 694649 individuals of European ancestry.Human molecular genetics 2019, 28:166-174.

26. Hübel C, Gaspar HA, Coleman JRI, Finucane H, Purves KL, Hanscombe KB, Prokopenko I, Graff M, Ngwa JS, Workalemahu T, et al: Genomics of body fat percentage may contribute to sex bias in anorexia nervosa.American journal of medical genetics Part B, Neuropsychiatric genetics : the official publication of the International Society of Psychiatric Genetics 2019, 180:428-438. 
27. Horikoshi M, Beaumont RN, Day FR, Warrington NM, Kooijman MN, Fernandez-Tajes J, Feenstra B, van Zuydam NR, Gaulton KJ, Grarup N, et al: Genome-wide associations for birth weight and correlations with adult disease.Nature 2016, 538:248-252.

28. Surakka I, Horikoshi M, Mägi R, Sarin AP, Mahajan A, Lagou V, Marullo L, Ferreira T, Miraglio B, Timonen S, et al: The impact of low-frequency and rare variants on lipid levels. Nature genetics 2015, 47:589-597.

29. Horikoshi M, Mägi R, van de Bunt M, Surakka I, Sarin AP, Mahajan A, Marullo L, Thorleifsson G, Hägg S, Hottenga JJ, et al: Discovery and Fine-Mapping of Glycaemic and Obesity-Related Trait Loci Using High-Density Imputation.PLoS genetics 2015, 11:e1005230.

30. Mahajan A, Taliun D, Thurner M, Robertson NR, Torres JM, Rayner NW, Payne AJ, Steinthorsdottir V, Scott RA, Grarup N, et al: Fine-mapping type 2 diabetes loci to single-variant resolution using highdensity imputation and islet-specific epigenome maps.Nature genetics 2018, 50:1505-1513.

31. Benyamin B, He J, Zhao Q, Gratten J, Garton F, Leo PJ, Liu Z, Mangelsdorf M, Al-Chalabi A, Anderson $\mathrm{L}$, et al: Cross-ethnic meta-analysis identifies association of the GPX3-TNIP1 locus with amyotrophic lateral sclerosis.Nature communications 2017, 8:611.

32. Yang J, Weedon MN, Purcell S, Lettre G, Estrada K, Willer CJ, Smith AV, Ingelsson E, O'Connell JR, Mangino $\mathrm{M}$, et al: Genomic inflation factors under polygenic inheritance. European journal of human genetics : EJHG 2011, 19:807-812.

33. Wang K, Li M, Hakonarson H: ANNOVAR: functional annotation of genetic variants from highthroughput sequencing data.Nucleic acids research 2010, 38:e164.

34. Andreassen OA, Thompson WK, Schork AJ, Ripke S, Mattingsdal M, Kelsoe JR, Kendler KS, O'Donovan MC, Rujescu D, Werge T, et al: Improved detection of common variants associated with schizophrenia and bipolar disorder using pleiotropy-informed conditional false discovery rate.PLOS genetics 2013, 9:e1003455.

35. Ramasamy A, Trabzuni D, Guelfi S, Varghese V, Smith C, Walker R, De T, Coin L, de Silva R, Cookson $M R$, et al: Genetic variability in the regulation of gene expression in ten regions of the human brain.Nature neuroscience 2014, 17:1418-1428.

36. Kamburov A, Pentchev K, Galicka H, Wierling C, Lehrach H, Herwig R: ConsensusPathDB: toward a more complete picture of cell biology.Nucleic acids research 2011, 39:D712-717.

37. Zhang L, Tang L, Huang T, Fan D: Life Course Adiposity and Amyotrophic Lateral Sclerosis: A Mendelian Randomization Study.Annals of neurology 2020, 87:434-441.

38. Wang D, Chan CC, Cherry S, Hiesinger PR: Membrane trafficking in neuronal maintenance and degeneration.Cellular and molecular life sciences : CMLS 2013, 70:2919-2934.

39. Kiral FR, Kohrs FE, Jin EJ, Hiesinger PR: Rab GTPases and Membrane Trafficking in Neurodegeneration.Current biology : CB 2018, 28:R471-r486.

40. Aoki Y, Manzano R, Lee Y, Dafinca R, Aoki M, Douglas AGL, Varela MA, Sathyaprakash C, Scaber J, Barbagallo $\mathrm{P}$, et al: C9orf72 and RAB7L1 regulate vesicle trafficking in amyotrophic lateral sclerosis and frontotemporal dementia.Brain : a journal of neurology 2017, 140:887-897. 
41. T S, U N, Y N, A K, C K, Y K, M K, T S, M N, B M, et al: Reduction rate of body mass index predicts prognosis for survival in amyotrophic lateral sclerosis: a multicenter study in Japan.Amyotrophic lateral sclerosis : official publication of the World Federation of Neurology Research Group on Motor Neuron Diseases 2012, 13:363-366.

42. JC D, PM P, TC T, JM V, D S, P C: Nutritional status is a prognostic factor for survival in ALS patients.Neurology 1999, 53:1059-1063.

43. Yang Q, Vijayakumar A, Kahn BB: Metabolites as regulators of insulin sensitivity and metabolism.Nature reviews Molecular cell biology 2018, 19:654-672.

44. Y D, Y W, X G, J H, W W, A H, Q F, P L, L L, X L, F Z: A Genome-wide Expression Association Analysis Identifies Genes and Pathways Associated with Amyotrophic Lateral Sclerosis. Cellular and molecular neurobiology 2018, 38:635-639.

45. Lan ZJ, Hu Y, Zhang S, Li X, Zhou H, Ding J, Klinge CM, Radde BN, Cooney AJ, Zhang J, Lei Z: GGNBP2 acts as a tumor suppressor by inhibiting estrogen receptor $a$ activity in breast cancer cells.Breast cancer research and treatment 2016, 158:263-276.

46. Hill-Burns EM, Ross OA, Wissemann WT, Soto-Ortolaza Al, Zareparsi S, Siuda J, Lynch T, Wszolek ZK, Silburn PA, Mellick GD, et al: Identification of genetic modifiers of age-at-onset for familial Parkinson's disease.Hum Mol Genet 2016, 25:3849-3862.

47. Gibson SB, Abbott D, Farnham JM, Thai KK, McLean H, Figueroa KP, Bromberg MB, Pulst SM, Cannon-Albright L: Population-based risks for cancer in patients with ALS.Neurology 2016, 87:289294.

48. Kim EK, Choi EJ: Pathological roles of MAPK signaling pathways in human diseases.Biochimica et biophysica acta 2010, 1802:396-405.

49. MR G, L M, F P: Dyslipidemia is a protective factor in amyotrophic lateral sclerosis.Neurology 2008, 71:956; author reply 956-957.

50. C I, L C, Y Z, J T, L Y, F F: Lipids, apolipoproteins, and prognosis of amyotrophic lateral sclerosis.Neurology 2020, 94:e1835-e1844.

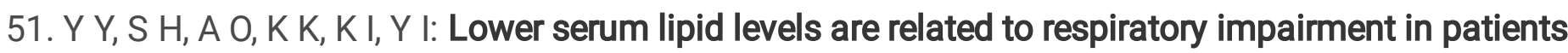
with ALS.Neurology 2010, 74:2027; author reply 2027-2028.

52. Jiang J, Zhu Q, Gendron TF, Saberi S, McAlonis-Downes M, Seelman A, Stauffer JE, Jafar-Nejad P, Drenner K, Schulte D, et al: Gain of Toxicity from ALS/FTD-Linked Repeat Expansions in C90RF72 Is Alleviated by Antisense Oligonucleotides Targeting GGGGCC-Containing RNAs.Neuron 2016, 90:535550.

53. Jääskeläinen O, Solje E, Hall A, Katisko K, Korhonen V, Tiainen M, Kangas AJ, Helisalmi S, Pikkarainen M, Koivisto A, et al: Low Serum High-Density Lipoprotein Cholesterol Levels Associate with the C9orf72 Repeat Expansion in Frontotemporal Lobar Degeneration Patients. Journal of Alzheimer's disease : JAD 2019, 72:127-137.

54. M S, PT vD, AE V, MH H, MH R, SW dJ, AJ vdK, M dV, NC V, JH V, LH vdB: Prior medical conditions and the risk of amyotrophic lateral sclerosis. Journal of neurology 2014, 261:1949-1956. 
55. MA K, RS R, RM S, O G, J H, MG W: Diabetes Mellitus, Obesity, and Diagnosis of Amyotrophic Lateral Sclerosis: A Population-Based Study.JAMA neurology 2015, 72:905-911.

56. QQ W, Y C, B C, RW O, L Z, Y H, X G, H S: Blood hemoglobin A1c levels and amyotrophic lateral sclerosis survival.Molecular neurodegeneration 2017, 12:69.

57. Marat AL, Dokainish H, McPherson PS: DENN domain proteins: regulators of Rab GTPases. The Journal of biological chemistry 2011, 286:13791-13800.

58. CA D, D C-M, K V, MJ S: The emerging role of guanine nucleotide exchange factors in ALS and other neurodegenerative diseases. Frontiers in cellular neuroscience 2014, 8:282.

59. TP L, RD D, AT G, LH W, MJ H: The product of C9orf72, a gene strongly implicated in neurodegeneration, is structurally related to DENN Rab-GEFs.Bioinformatics (Oxford, England) 2013, 29:499-503.

\section{Tables}

Table 1

Shared risk loci between ALS and obesity-related traits.

\begin{tabular}{|c|c|c|c|c|c|c|c|}
\hline SNP & $\begin{array}{l}\text { Genomic } \\
\text { position } \\
\text { (GRCh37) }\end{array}$ & $\begin{array}{l}\text { Closest } \\
\text { gene }\end{array}$ & A1 & $\begin{array}{l}\text { Associated } \\
\text { phenotype }\end{array}$ & $\begin{array}{l}\text { FDR } \\
\text { value }\end{array}$ & $\begin{array}{l}\text { Original } \\
\text { ALS } \\
\text { P value }\end{array}$ & $\begin{array}{l}\text { Replication } \\
\text { ALS } \\
\text { P value }\end{array}$ \\
\hline rs62333164 & $4: 170583157$ & CLCN3 & $A$ & BMI & 0.0386 & $\begin{array}{l}5.24 \mathrm{E}- \\
06\end{array}$ & 3.84E-06 \\
\hline rs3849942 & 9:27543281 & C9orf72 & $\mathrm{T}$ & HDL-C,LDL-C & 0.0329 & $\begin{array}{l}5.11 \mathrm{E}- \\
30\end{array}$ & 1.20E-22 \\
\hline rs170663 & 14:31049409 & G2E3 & $\mathrm{T}$ & BMI & 0.0269 & $\begin{array}{l}5.64 \mathrm{E}- \\
07\end{array}$ & $2.22 \mathrm{E}-06$ \\
\hline rs8018993 & 14:31138219 & SCFD1 & $A$ & $\mathrm{BMI}$ & 0.0223 & $\begin{array}{l}2.02 \mathrm{E}- \\
06\end{array}$ & 8.00E-06 \\
\hline rs11160036 & $14: 92481047$ & TRIP11 & $A$ & BMI & 0.0238 & $\begin{array}{l}1.38 \mathrm{E}- \\
06\end{array}$ & $1.50 \mathrm{E}-04$ \\
\hline rs978220 & $14: 92558135$ & ATXN3 & $\mathrm{T}$ & $\mathrm{BMI}$ & 0.0231 & $\begin{array}{l}1.66 \mathrm{E}- \\
06\end{array}$ & 2.61E-05 \\
\hline rs12603276 & $17: 34946547$ & GGNBP2 & $\mathrm{T}$ & BMI,WHR,BFP & 0.0259 & $\begin{array}{l}6.06 \mathrm{E}- \\
05\end{array}$ & $9.21 \mathrm{E}-06$ \\
\hline rs1976704 & $17: 76401318$ & PGS1 & $\mathrm{T}$ & HDL-C & 0.0384 & $\begin{array}{l}1.50 \mathrm{E}- \\
04\end{array}$ & $2.38 \mathrm{E}-04$ \\
\hline rs68069258 & $22: 50748930$ & DENND6B & C & $\mathrm{T} 2 \mathrm{D}$ & 0.0454 & $\begin{array}{l}6.00 \mathrm{E}- \\
06\end{array}$ & $2.89 \mathrm{E}-02$ \\
\hline
\end{tabular}


Table 2

eQTL revealing functional effects of shared risk SNPs in human brain tissue.

\begin{tabular}{|lllll|}
\hline Genomic position (GRCh37) & SNP & Closest gene & eQTL & \\
\cline { 4 - 5 } & & & Gene & P value \\
\hline $9: 27511593$ & rs1845699 & C9orf72 & TEK & $3.40 \mathrm{E}-05$ \\
\hline $14: 92525145$ & rs1047795 & ATXN3 & SLC24A4 & $1.10 \mathrm{E}-03$ \\
\hline $14: 92558135$ & rs978220 & ATXN3 & ATXN3 & $1.10 \mathrm{E}-06$ \\
\hline 17:34917608 & rs6607326 & GGNBP2 & MYO19 & $3.10 \mathrm{E}-04$ \\
\hline $17: 34946547$ & rs12603276 & GGNBP2 & GGNBP2 & $8.40 \mathrm{E}-12$ \\
\hline $17: 76395421$ & rs2292643 & PGS1 & PGS1 & $6.60 \mathrm{E}-11$ \\
\hline $17: 76395421$ & rs2292643 & PGS1 & USP36 & $2.00 \mathrm{E}-04$ \\
\hline
\end{tabular}

Figures 

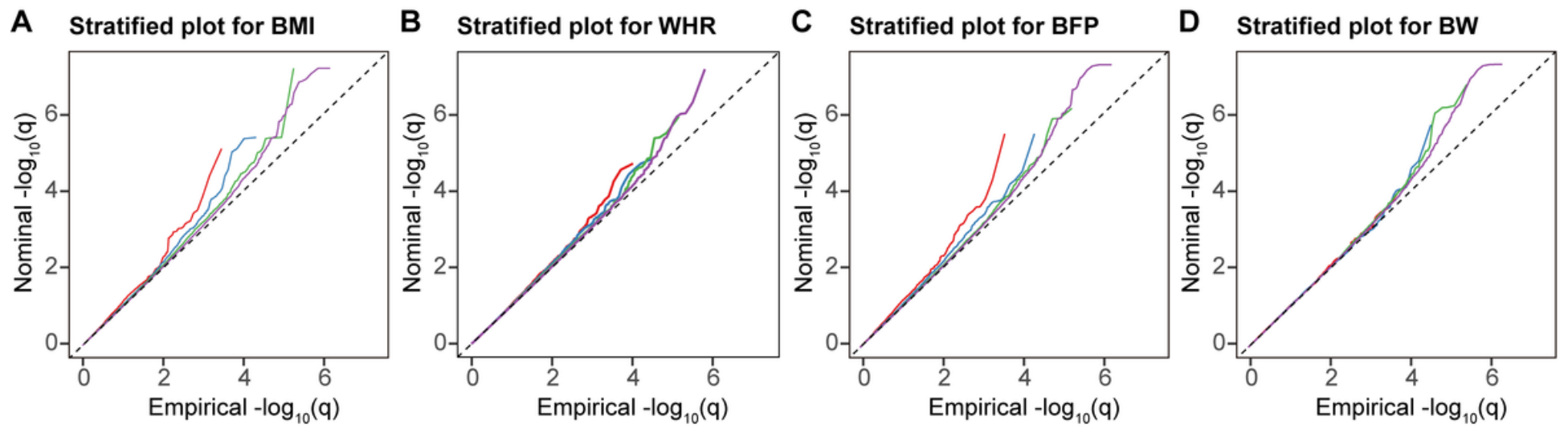

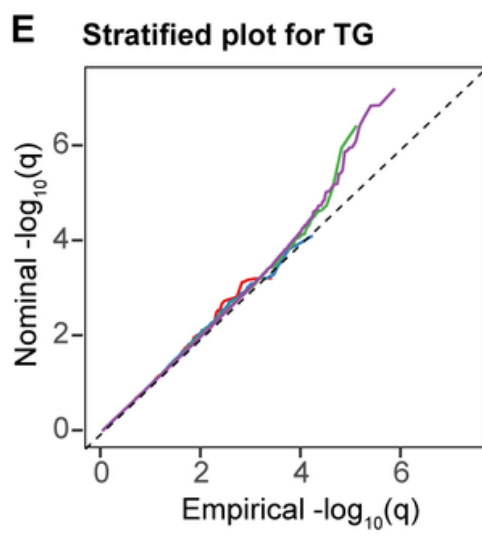

F Stratified plot for TC

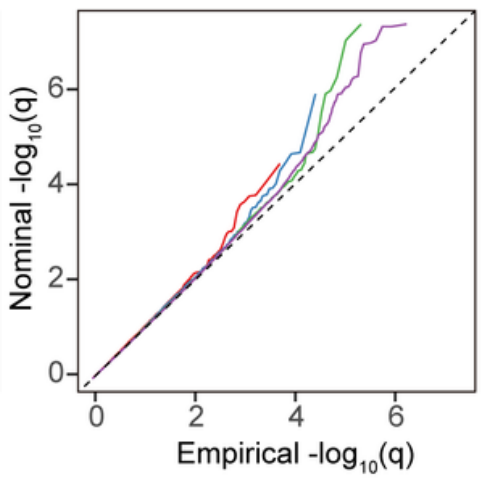

G Stratified plot for LDL-C

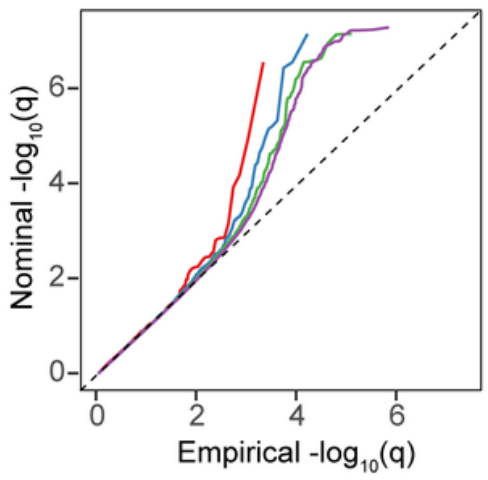

K Stratified plot for FI

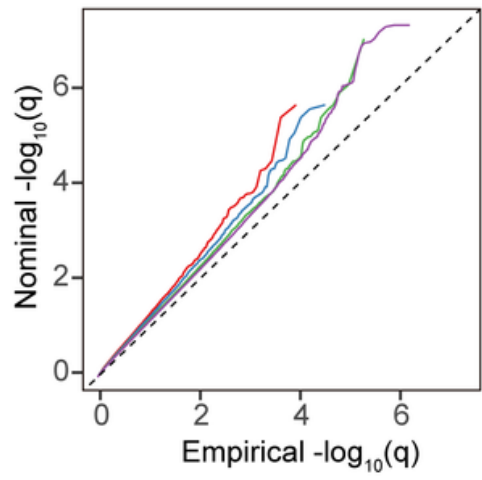

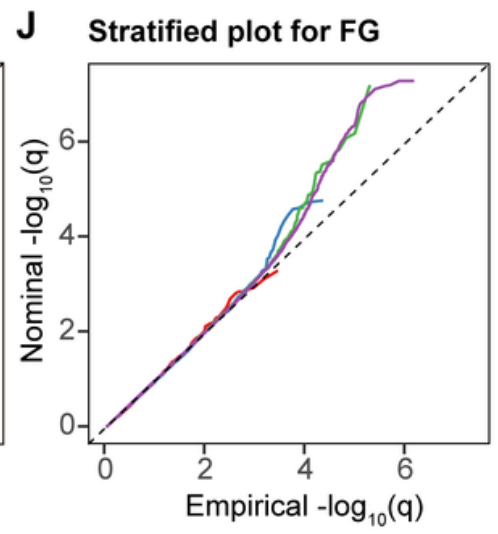

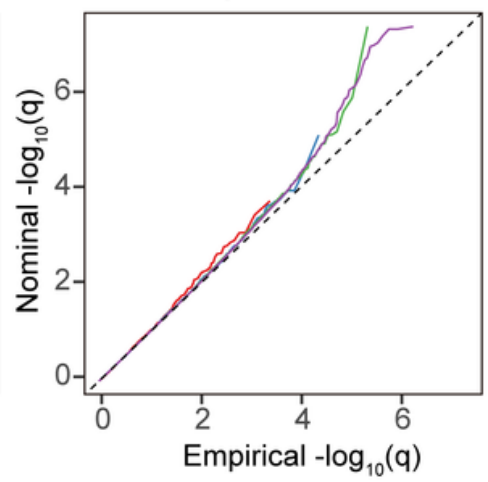

H Stratified plot for HDL-C

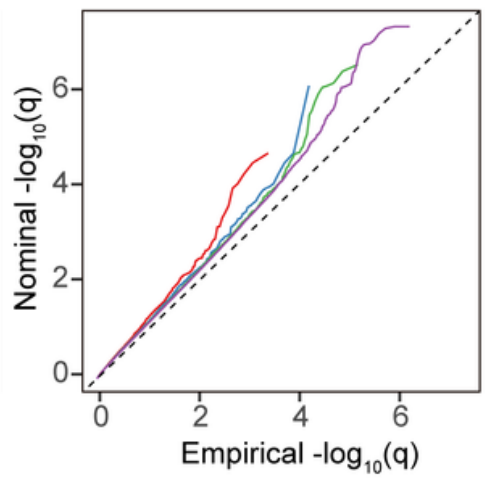

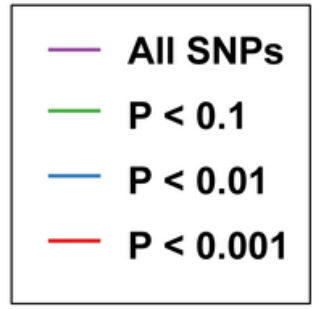

Figure 1

Enrichment plots. Conditional quantile-quantile plots of nominal versus empirical -log10(P) of ALS as a function of significance of association with obesity-related traits. Dashed lines indicate the null hypothesis. 


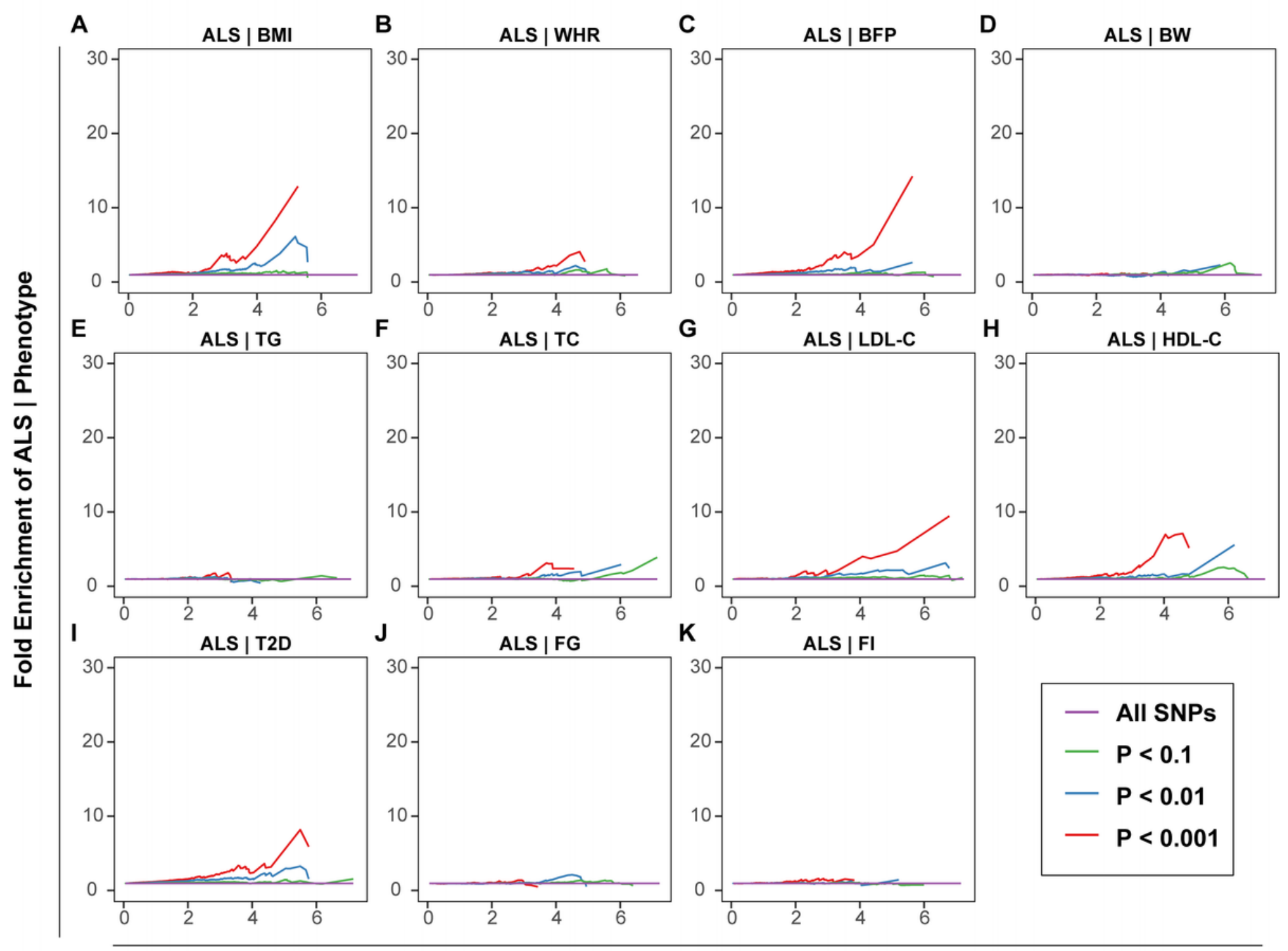

Nominal $-\log _{10} P$-value in ALS

Figure 2

Fold-enrichment plot. Fold-enrichment plot of nominal - $\log 10(P)$ of ALS as a function of significance of association with obesity-related traits. 


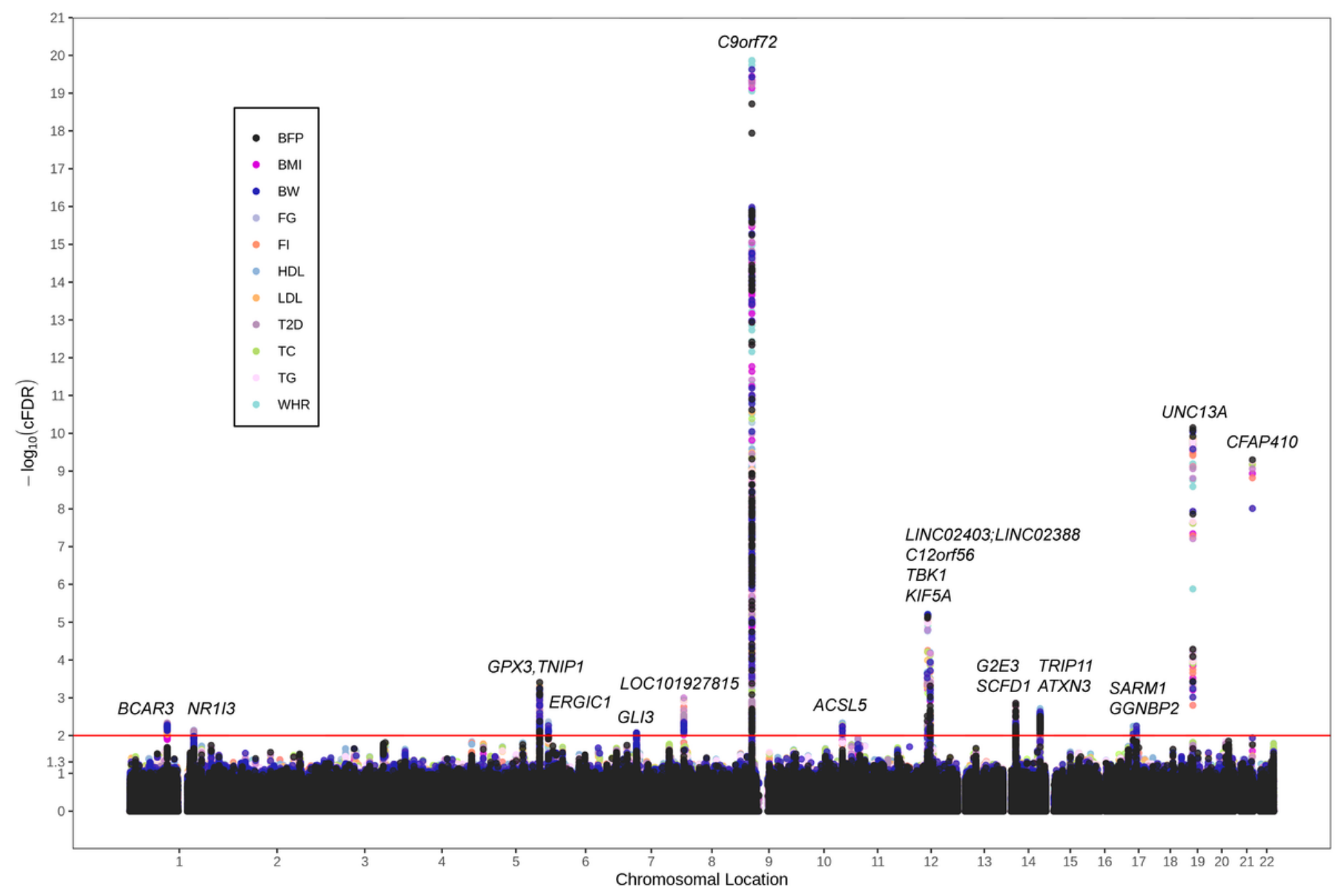

Figure 3

Conditional Manhattan plots. Conditional Manhattan plots showing risk loci for ALS conditional on each obesity-related trait. The dotted horizontal line represents the significant threshold (conditional FDR = 0.01). 


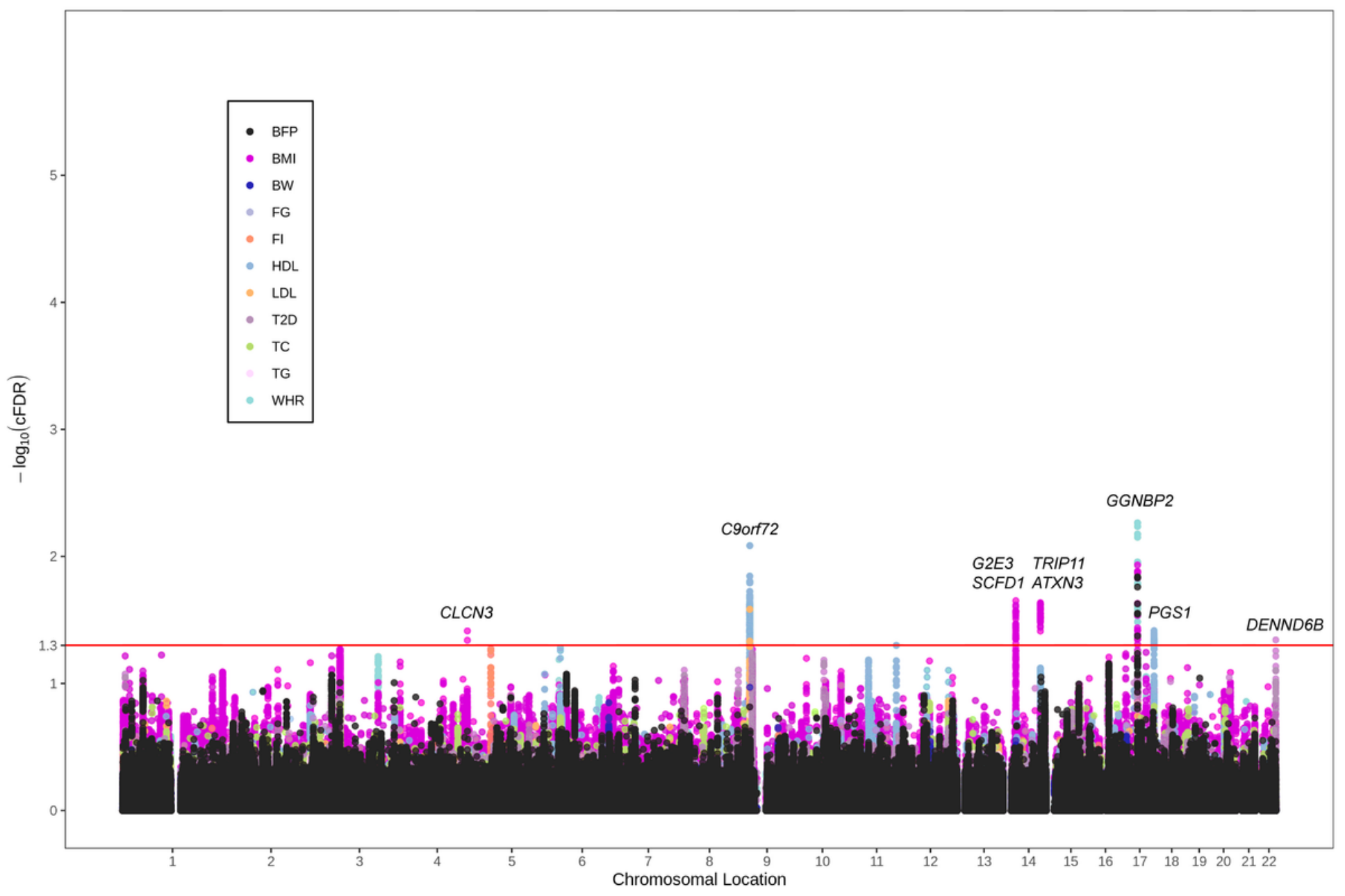

Figure 4

Conjunctional Manhattan plots. Conjunctional Manhattan plots showing shared genetic loci between ALS and each obesity-related trait. The dotted horizontal line represents the significant threshold (conjunctional FDR $=0.05$ ).

\section{Supplementary Files}

This is a list of supplementary files associated with this preprint. Click to download.

- SupplementaryMaterial.pdf 